O. R. Abib

Nagoya Math. J.

Vol. 73 (1979), 29-40

\title{
EQUATIONS DE LIE INVARIANTES PAR UN PSEUDOGROUPE DE LIE
}

\author{
ODINETE RENÉE ABIB
}

\section{§ 0. Introduction}

Soit $(\mathscr{L}, M)$ un pseudogroupe de Lie infinitésimal [4] transitif d'ordre $h$ sur une variété $M$ différentiable, $J^{h} \mathscr{L}$ son équation de définition et $L$ son algèbre formelle au point $a$ de $M$.

Dénotons par $I(L)$ l'ensemble des idéaux fermés de $L$ et par $I\left(J^{h} \mathscr{L}\right)$ l'ensemble des équations différentielles $R^{m} \subset J^{m} \mathscr{L}, m \geq h$, invariantes par $\Gamma$ et formellement intégrables, $\Gamma$ désignant le pseudogroupe de transformations associé à $\mathscr{L}$. L'un des buts de ce papier est de définir une application surjective de $I\left(J^{h} \mathscr{L}\right)$ dans $I(L)$. La surjectivité implique en particulier que tout idéal fermé de $L$ est l'espace des solutions formelles en $a$ d'une équation de Lie appartenant à $I\left(J^{h} \mathscr{L}\right),(\S 2, \S 3)$. En plus, dans le cas analytique, cette équation est l'équation de définition d'un sous-pseudogroupe normal de $\mathscr{L}$ (Corollaire 2.2).

D'autre part, $F(L)$ étant l'ensemble des idéaux fermés de $L$ définis par un feuilletage $(\S 2), F\left(J^{h} \mathscr{L}\right)$ l'ensemble des équations différentielles $R^{m}$ de $I\left(J^{h} \mathscr{L}\right)$ pour lesquelles $\Pi_{0}\left(R^{m}\right)=E$ soit un sous-fibré intégrable de $T(M)$ invariant par l'action de $\mathscr{L}$ et $L \cap J_{a}^{\infty} E=\lim _{\ell}\left(R_{a}^{m}\right)^{+\ell}$, nous montrons (§3) que l'application précédente envoie $F\left(J^{h} \mathscr{L}\right)$ sur $F(L)$.

Etant donné $I$ un idéal fermé de $L$ on lui associe toujours une algèbre de Lie normale tronquée d'ordre $h \geq 1$ (§4). Inversement, nous montrons (Théorème 4.1) que à toute algèbre de Lie $W^{h}$ normale tronquée d'ordre $h \geq 1$, satisfaisant certaines conditions cohomologiques, correspond un idéal fermé $I$ de $L$ ayant $W^{h}$ comme algèbre tronquée d'ordre $h$. En plus $I$ est unique à un isomorphisme près. Ce résultat généralise ainsi le Théorème 6 de [1] pour le cas non-transitif.

Il est à signaler que certains de nos résultats semblent concorder avec ceux obtenus indépendamment par H. Goldschmidt dans un tout

Received September 3, 1977. 
autre formalisme (cf. [2], §10).

\section{§1. Notations}

Etant donné $T=T(M)$ le fibré tangent d'une variété différentiable $M, J^{k} T(M)$ désigne l'ensemble de tous les jets d'ordre $k$ des sections $d u$ fibré vectoriel $T$ et $\underline{J^{k} T(M)}$ le faisceau des sections de $J^{k} T(M)$ qui est un faisceau de $R$-algèbre de Lie [4]:

$$
\underline{J^{k} T} \times \underline{J^{k} T} \rightarrow \underline{J^{k} T},
$$

$\left[f \cdot j^{k} X, g \cdot j^{h} Y\right]=f \cdot g j^{h}[X, Y]+f(X \cdot g) j^{k} Y-g(Y \cdot f) j^{h} X$, où $[X, Y]$ est le crochet de Lie des champs de vecteurs $X$ et $Y, f$ et $g$ sont des fonctions différentiables numériques.

Ce crochet induit un morphisme de fibrés vectoriels sur $M$, à savoir:

$$
\begin{aligned}
{[,]^{\prime}: J^{h+1} T \times J^{h+1} T } & \rightarrow J^{h} T \\
\left(j^{h+1} X, J^{h+1} Y\right) & \rightarrow j^{h}[X, Y] .
\end{aligned}
$$

Considérons $D: \underline{J^{h+1} T} \rightarrow \underline{T^{*} \otimes J^{h} T}$ l'opérateur de Spencer défini par $D\left(f \cdot j^{n+1} X\right)=d f \otimes j^{n} X$.

LEMME 1.1. (a) $\left[f \cdot j^{h} X, g \cdot j^{h} Y\right]=\left[f \cdot j^{h+1} X, g \cdot j^{h+1} Y\right]^{\prime}+D\left(g \cdot j^{h+1} Y\right)$ $\cdot(f \cdot X)-D\left(f \cdot j^{h+1} X\right)(g \cdot Y)$.

(b) $D\left(\left[f \cdot j^{h+1} X, g \cdot j^{h+1} Y\right]^{\prime}\right)(Z)=\left[D\left(f \cdot j^{h+1} X\right)(Z), g \cdot j^{h} Y\right]^{\prime}+\left[f \cdot j^{h+1} X\right.$, $\left.D\left(g \cdot j^{h+1} Y\right)(Z)\right]^{\prime}$.

La démonstration de ce résultat découle des définitions précédentes.

Désignons par $I I$ la projection naturelle de $J^{h+1} T$ sur $J^{h} T$; soit $a \in M$ et $J_{a}^{\infty} T$ la limite projective relative à ce morphisme des $J_{a}^{h} T$. Le crochet $[,]^{\prime}$ définit alors sur cet ensemble une structure d'algèbre de Lie de dimension infinie.

Etant donné un pseudogroupe de Lie infinitésimal transitif $\mathscr{L}$ sur $M$, dénotons par $J^{h} \mathscr{L}$ le fibré vectoriel de tous les jets d'ordre $h$ des sections locales de $\mathscr{L}$ dont le faisceau des sections $\underline{J^{h} \mathscr{L}}$ est un sousfaisceau du faisceau d'algèbre de Lie $J^{h} T$. En particulier, le crochet $[,]^{\prime}$ induit le crochet de $\underline{J^{h} \mathscr{L}}$ à valeurs dans $\underline{J^{h-1} \mathscr{L}}$ :

$$
[,]^{\prime}: \underline{J^{h} \mathscr{L}} \times \underline{J^{h} \mathscr{L}} \rightarrow \underline{J^{h-1} \mathscr{L}} .
$$

Désignons par $L \subset J_{a}^{\infty} T$ la limite projective des $J_{a}^{h} \mathscr{L}$ par rapport à la projection $\Pi ; L$ est une sous-algèbre de Lie de $J_{a}^{\infty} T$ qui sera dite 
l'algèbre formelle de $\mathscr{L}$ en $a \in M$. De plus $L$ est une algèbre de Lie filtrée :

$$
L=L_{-1} \supset L_{0} \supset L_{1} \supset \ldots \supset L_{h} \supset \cdots
$$

où $L_{h}$ est le noyau de la projection $\Pi_{h}$ de $L$ sur $J_{a}^{h} \mathscr{L}$. Posons $g^{h}=L_{h-1} / L_{h}$, pour $h \geq 0$. On a naturellement les suites exactes:

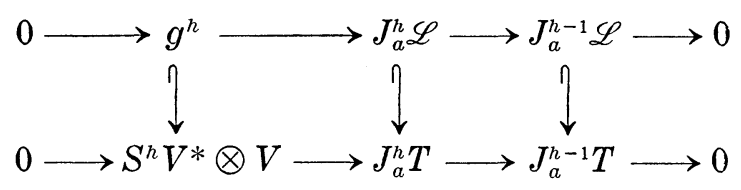

avec $V=T_{a}(M)=L / L_{0}$.

\section{$\S 2$.}

Soit $I$ un idéal fermé de l'algèbre formelle $L$ de $\mathscr{L}$ en $a \in M$. Posons $I_{h}=I \cap L_{h}$ pour $h \geq-1$ de sorte que $p^{h}=I_{h-1} / I_{h}$ s'identifie à un sous-espace de $g^{h}$; alors,

LEMME 2.1. (a) $\left[I^{h}, J_{a}^{h} \mathscr{L}\right]^{\prime} \subset I^{h-1}$.

(b) $\left[p^{h}, J^{h} \mathscr{L}\right]^{\prime} \subset p^{h-1}$.

(c) $\left[g^{h}, g^{h}\right]^{\prime}=\{0\}$.

ò̀ $I^{h}=I / I_{h}$.

La deuxième inclusion et la transitivité de $\mathscr{L}$ nous donnent une injection :

$$
\delta: p^{h+1} \rightarrow V^{*} \otimes p^{h},
$$

$\delta(X)(v)=[X, y]^{\prime}$ où y est un élément de $J_{a}^{h+1} \mathscr{L}$ qui se projette sur $v$; d'où une application

$$
\delta: \wedge^{j} V^{*} \otimes p^{h+1} \rightarrow \wedge^{j+1} V^{*} \otimes p^{h},
$$

défini par:

$$
\delta(f)\left(v_{1}, v_{2}, \cdots, v_{j+1}\right)=\sum_{s=1}^{j+1}(-1)^{j+1-s} f\left(v_{1}, v_{2}, \cdots, \hat{v}_{s}, \cdots, v_{j+1}\right) \cdot v_{s}
$$

pour $v_{1}, \cdots, v_{j+1}$ dans $V$.

On obtient ainsi un complexe:

$$
0 \longrightarrow p^{h} \stackrel{\delta}{\longrightarrow} V^{*} \otimes p^{h-1} \stackrel{\delta}{\longrightarrow} \wedge^{2} V^{*} \otimes p^{h-2} \longrightarrow \cdots
$$


dont le groupe de cohomologie dans $\wedge^{j} V^{*} \otimes p^{h}$ sera dénoté par $H^{j, h}(I)$.

LEMME 2.2. Soit $(\mathscr{L}, M)$ un pseudogroupe de Lie infinitésimal transitif sur $M$ et $I$ un idéal fermé de $L$; alors il existe un entier $h_{0}$ tel que $H^{j, h}(I)=0$ pour tous $j \geq 0$ et $h \geq h_{0}$.

Preuve. Ce résultat résulte du fait que $M=\prod_{h \geq 0}\left(p^{h}\right)^{*}$ est un $S(V)$ module gradué de type fini.//

Définition 2.1. Un idéal fermé $I$ de $L$ est défini par un feuilletage dans $\left(L, L_{0}\right)$ si le seul idéal $J$ de $L$ vérifiant $I \subset J \subset I+L_{0}$ est $I$ luimême.

Exemple. Soit $E$ un sous fibré vectoriel integrable de $T(M)$ tel que $[E, \mathscr{L}] \subset \mathscr{L}$ et dénotons par $J_{a}^{\infty} E$ l'algèbre formelle en a associée à $E$; alors $I=L \cap J_{a}^{\infty} E$ est un idéal fermé de $L$ défini par un feuilletage dans $\left(L, L_{0}\right)$; (cf. démonstration du Théorème 2.1. (5.d)).

Remarque. Soient $J$ et $I$ deux idéaux de $L$ définis par un feuilletage dans $\left(L, L_{0}\right)$ tels $\Pi_{0}(I)=\Pi_{0}(J)$ où $\Pi_{0}: L \rightarrow T_{a}(M)$ est la projection canonique; alors $I=J$ : en effet, $\Pi_{0}(I)=\Pi_{0}(J)$ entraîne $I \subset J+L_{0}$ et $J \subset I$ $+L_{0}$. On a $I \subset I+J \subset I+L_{0}$ et $I+J$ est un idéal fermé de $L$; d'où, $I \subset I+J$; ainsi $J \subset I$; de même, $I \subset J$; d'où, $I=J . / /$.

Proposition 2.1. Soit I un idéal fermé de l'algèbre formelle $L$. Considérons $h_{0} \geq 0$ un entier tel que $H^{1, h}(I)=0$ pour $h \geq h_{0}$; alors $I$ est un idéal défini par un feuilletage dans $\left(L, L_{h_{0}}\right)$.

Preuve. $\quad H^{1, h}(I)=0$ entraîne que $p^{h+1}$ coïncide avec le premier prolongement $\left(p^{h}\right)^{+1}$ de $p^{h}$. Soit $J$ un idéal fermé de $L$ tel que $I \subset J \subset L$ $+L_{h_{0}} ;$ alors $I / I_{h_{0}}=J / J_{h_{0}}$ et $p^{h_{0}}=q^{h_{0}}=J_{h_{0}-1} / J_{h_{0}}$; pour $h \geq h_{0}, q^{h+1} \subset\left(q^{h}\right)^{+1}$ $=\left(p^{h}\right)^{+1}=p^{h+1}$; donc, $q^{h+1}=p^{h+1}$ pour $h \geq h_{0}$. Ainsi $I / I_{h}=J / J_{h}$, pour $h \geq h_{0}$; d'où, $J \subset I+I_{h} \subset I+L_{h}$, pour $h \geq h_{0}$; d'où, $J \subset \bigcap_{h \geq h_{0}}\left(I+L_{h}\right)=I$ car $I$ est fermé; ainsi on obtient $I=J$. e.q.f.d.

THÉORÈme 2.1. Soit L l'algèbre formelle d'un pseudogroupe de Lie infinitésimal transitif $(\mathscr{L}, M)$ et $I$ un idéal fermé de $L$.

(1) Pour tout $h \geq 1$ il existe une équation de Lie $R^{h} \subset J^{h} \mathscr{L}$ telle que

$$
R_{a}^{h}=I / I_{h}=\Pi_{h}(I) \text {. }
$$

(2) Si $R^{h+1}$ est l'équation de Lie qui correspond à $\Pi_{h+1}(I)$, on $a$ : 
(a) $\left[\underline{R^{h+1}}, \underline{J^{h+1} \mathscr{L}}\right]^{\prime} \subset R^{h}$.

(b) $R^{h+1} \subset\left(R^{h}\right)^{+1}$ le premier prolongement de $R^{h}$.

(3) $\left[\underline{R^{h}}, \underline{J^{h} \mathscr{L}}\right] \subset \underline{R^{h}}$.

(4) Soit $h_{0}$ l'entier tel que $H^{1, h}(I)=0$ pour $h \geq h_{0}$; alors $R^{h_{0}}$ est formellement intégrable et $R^{h_{0}+\ell}$ est le $\ell$-ième prolongement $\left(R^{h_{0}}\right)^{+\ell}$ de $R^{h_{0}}$ pour $\ell \geq 0$. De plus, I coîncide avec $\lim _{h}\left(R_{a}^{h}\right)$.

(5) Il existe un sous-fibré vectoriel $E$ de $T$ tel que:

(a) $E_{a}=\Pi_{0}(I)$.

(b) $[\underline{E}, \mathscr{L}] \subset \mathscr{L}$, Ceci entraîne $[\underline{E}, \underline{E}] \subset \underline{E}$.

(c) $R^{h} \subset J^{h} E$, pour $h \geq 1$.

(d) I est défini par un feuilletage dans $\left(L, L_{0}\right)$ si, et seulement si, $I=L \cap J_{a}^{\infty} E$.

Preuve. Montrons d'abord l'existence de $E \subset T(M)$. L'inclusion, $\left[g^{1}, I / I_{1}\right]^{\prime} \subset I / I_{0}$ entraîne $I / I_{0}$ sous-espace de $L / L_{0}=V$ invariant par $g^{1}$. Ceci entraîne $I / I_{0}$ invariant par le groupe d'isotropie $G^{1}$ du pseudogroupe de transformations $\Gamma$ associée à $\mathscr{L}$; donc, $\Gamma$ déplace $I / I_{0}$ pour définir un sous-fibré vectoriel $E$ de $T(M)$; par construction on a $[\underline{E}, \mathscr{L}] \subset \mathscr{L}$; $[\underline{E}, \underline{E}] \subset \underline{E}$ : en effet, soient $X, Y$ deux sections locales de $E$ au voisinage $U$ d'un point $x$ de $M$; soient $j_{x}^{1} X^{\prime}, j_{x}^{1} Y^{\prime}$ dans $R^{1}$ tels que $X_{x}^{\prime}=X_{x}$ et $Y_{x}^{\prime}$ $=Y_{x} ;$ alors,

$\left[j_{x}^{1} X^{\prime}, j_{x}^{1} Y^{\prime}\right]^{\prime}=\left[X^{\prime}, Y^{\prime}\right](x)$ est dans $E_{x} ; \quad$ On a $[X, Y]=\left[X-X^{\prime}, Y-Y^{\prime}\right]$ $+\left[X, Y^{\prime}\right]+\left[X^{\prime}, Y\right]-\left[X^{\prime}, Y^{\prime}\right] ; \quad$ En $x$, le deuxiéme membre est dans $E_{x}$; d'oú, le resultat; En plus, $\left[J_{a}^{h+1} \mathscr{L} \cap J_{a}^{h+1} E, J_{a}^{h+1} \mathscr{L}\right] \subset J_{a}^{h} \mathscr{L} \cap J_{a}^{h} E$ pour $h \geqslant 0$; ainsi $L \cap J_{a}^{\infty} E$, est un ideal de $L$.

La construction de $R^{h}$ est presque analogue; l'inclusion, [ $J_{a}^{h+1} \mathscr{L}$, $\left.\Pi_{h+1}(I)\right]^{\prime} \subset \Pi_{h}(I)$ implique que $\Pi_{h}(I)$ est invariant par le groupe d'isotropie d'ordre $h$ de $\Gamma$; ainsi $\Gamma$ déplace $\Pi_{h}(I)$ pour définir un sous-fibré vectoriel $R^{h}$ de $J^{h} T(M)$. Par construction, $R^{h} \subset J^{h} \mathscr{L}$ pour $h \geq 1, R^{h+1}$ se projecte sur $R^{h},\left[\underline{R^{h+1}}, \underline{J^{h+1} \mathscr{L}}\right]^{\prime} \subset \underline{R^{h}}$ et $\left[\underline{R^{h}}, \underline{J^{h} \mathscr{L}}\right] \subset \underline{R^{h}}$ pour $h \geq 1$; d'où, $R^{h}$ est une équation de Lie d'ordre $h$ sur $T(M)$. De plus, la projection $\Pi: J^{1} \mathscr{L} \rightarrow T(M)$ envoie $R^{1}$ sur $E$ et $R_{a}^{1} \subset J_{a}^{1} E$. Par invariance on obtient $R^{1} \subset J^{1} E . \quad$ Supposons $R^{h+1} \subset\left(R^{h}\right)^{+1}$; alors si $R^{h} \subset J^{h} E$ on a $R^{h+1} \subset\left(R^{h}\right)^{+1}$ $\subset\left(J^{h} E\right)^{+1}=J^{h+1} E$; d'où, $R^{h} \subset J^{h} E$ pour tout $h \geq 1$.

Montrons que $R^{h+1} \subset\left(R^{h}\right)^{+1}$. Soit $\xi^{h+1}=f \cdot j^{h+1} X$ une section de $R^{h+1}$ et $Y$ dans $\mathscr{L}$; alors, 


$$
\left[f \cdot j^{n} X, j^{n} Y\right]=\left[f \cdot j^{n+1} X, j^{h+1} Y\right]^{\prime}-D\left(f \cdot j^{h+1} X\right)(Y) ;
$$

ainsi ; $D\left(\xi^{h+1}\right)(Y)$ est une section de $R^{h}$. On obtient que $D$ envoie $R^{h+1}$ dans $\underline{T^{*} \otimes R^{h}}$ et $R^{h+1}$ se projecte sur $R^{h}$; d'où, $R^{h+1} \subset\left(R^{h}\right)^{+1}$ par la caractérisation de prolongement d'équation différentielle.

Montrons (4). Soit $h_{0}$ l'entier tel que $p^{h+1}=\left(p^{h}\right)^{+1}$ pour $h \geq h_{0}$ (d'après le Lemme 2.2., $h_{0}$ existe toujours). Si $t^{h}$ est le fibré vectoriel noyau de $\Pi: R^{h} \rightarrow R^{h-1}$ alors $t_{a}^{h}=p^{h}$ pour $h \geq 1$. On a $t_{a}^{h_{0}+1}=p^{h_{0}+1}=\left(p^{h_{0}}\right)^{+1}=\left(t_{a}^{h_{0}}\right)^{+1}$. De même, pour $\ell \geq 0$ on obtient, $t_{a}^{h_{0}+\ell}=\left(t_{a}^{h_{0}}\right)^{+\ell} ;\left(t^{h_{0}}\right)^{+\ell}$ est un fibré vectoriel sur $M$ et $t^{h_{0}+\ell} \subset\left(t^{h_{0}}\right)^{+\ell}$ car $R^{h_{0}+\ell}$ est contenu dans $\left(R^{h_{0}}\right)^{+\ell}$ d'après $(2 . \mathrm{b})$; ainsi, $t^{h_{0}+\ell}=\left(t^{h_{0}}\right)^{+\ell}$ pour tout $\ell \geq 0$; d'où, $\left(R^{h_{0}}\right)^{+1}=R^{h_{0}+1}$. Si $R^{h_{0}+\ell-1}$ $=\left(R^{h_{0}}\right)^{+\ell-1}$ on obtient $\left(R^{h_{0}}\right)^{+\ell}=\left(R^{h_{0}}\right)^{+\ell}$ pour $\ell \geq 0$. Nous avons donc démontré (4).

Démonstration de (5.d). $\quad L \cap J_{a}^{\infty} E$ est un idéal fermé de $L$ qui contient $I$ car $R^{h} \subset J^{h} E$ pour $h \geq 1$; en plus, $\Pi_{0}\left(L \cap J_{a}^{\infty} E\right)=I / I_{0}=E_{a}$; donc,

$$
I \subset L \cap J_{a}^{\infty} E \subset I+L_{0}
$$

Si $I$ est défini par un feuilletage dans $\left(L, L_{0}\right)$ on a $I=L \cap J_{a}^{\infty} E$. Réciproquement, supposons $L \cap J_{a}^{\infty} E=I$ et soit $J$ un idéal fermé de $L$ tel que,

$$
L \cap J_{a}^{\infty} E \subset J \subset L \cap J_{a}^{\infty} E+L_{0}
$$

alors $\Pi_{0}\left(L \cap J_{a}^{\infty} E\right)=\Pi_{0}(J)$. Soit $\left\{S^{h}\right\}_{h \geq 1}$ la suite d'équations de Lie qui correspond à $J$ et $F$ le sous-fibré intégrable de $T(M)$. On a $F_{a}=E_{a}$, $S^{h} \subset\left(J^{h} F \cap J^{h} \mathscr{L}\right)$ pour $h \geq 1$, et $E \subset F$. Ainsi $E=F$ et $J=\lim _{h} S_{a}^{h}$ $\subset J_{a}^{\infty} F \cap L=J_{a}^{\infty} E \cap L=I$; d'où, $I=J$; ce qui implique que $I$ est défini par un feuilletage dans $\left(L, L_{0}\right)$. c.q.f.d.

Corollaire 2.1. Soit $(\mathscr{L}, M)$ un pseudogroupe de Lie infinitésimal transitif sur $M$ et $I \subset L$ un idéal fermé de son algèbre formelle en $a$; alors il existe un entier $h_{0} \geq 0$ et une équation de Lie formellement intégrable $R^{h_{0}} \subset J^{h_{0}} \mathscr{L}$ telle que

$$
\left[\underline{R^{h_{0}}}, \underline{J^{h_{0}} \mathscr{L}}\right] \subset \underline{R^{h_{0}}}
$$

et 


$$
I=\lim _{\ell}\left(R_{a}^{h^{0}}\right)^{+\ell} .
$$

COROLlaire 2.2 [4]. Etant donnés un pseudogroupe de Lie infinitésimal analytique transitif $\mathscr{L}$ défini sur une variété analytique réelle $M$ et I un idéal fermé de $L$, il existe un sous-pseudo-groupe infinitésimal $\mathscr{L}_{0} \subset \mathscr{L}$ défini sur $M$ tel que

$$
\left[\mathscr{L}_{0}, \mathscr{L}\right] \subset \mathscr{L}_{0} \text { et } \lim _{h} J_{a}^{h} \mathscr{L}_{0}=I .
$$

Preuve. Considérons l'équation de Lie $R^{h_{0}}$ formellement intégrable donnée par le Corollaire 2.1. Soit $\mathscr{L}_{0}$ l'ensemble des solutions de $R^{h_{0}}$; alors $J^{h_{0}} \mathscr{L}_{0}=R^{h_{0}}$ et $R^{h_{0+\ell}}=\left(R^{h_{0}}\right)^{+\ell}=J^{h_{0}+\ell} \mathscr{L}_{0}, \forall \ell \geq 0$. Soit $X$ dans $\mathscr{L}_{0}$ et $Y$ dans $\mathscr{L}$; de $j^{h_{0}}[X, Y]=\left[j^{h_{0}} X, j^{h_{0}} Y\right] \in\left[\underline{R^{h_{0}}}, J^{h_{0}} \mathscr{L}\right] \subset \underline{R^{h_{0}}}$ on obtient $[X, Y] \in \mathscr{L}_{0} ;$ d'où $\left[\mathscr{L}_{0}, \mathscr{L}\right] \subset \mathscr{L}_{0}$. Ainsi $\mathscr{L}_{0}$ est un pseudo-groupe infinitésimal vérifiant le corollaire. c.q.f.d.

\section{$\S 3$.}

Les notations étant celles des paragraphes précédents, soit $h$ un entier tel que $J^{m+1} \mathscr{L}=\left(J^{m} \mathscr{L}\right)^{+1}$ pour $m \geq h$. Donc, $J^{h} \mathscr{L}$ est une équation de Lie transitive et formellement intégrable. Dénotons par $I(L)$ l'ensemble des idéaux fermés de $L$ et par $I\left(J^{h} \mathscr{L}\right)$ l'ensemble des équations différentielles $R^{m} \subset J^{m} \mathscr{L}$ formellement intégrable d'ordre $m, m \geq h$ telle que $\left[\underline{R^{m}}, \underline{J^{m} \mathscr{L}}\right] \subset \underline{R^{m}}$. Nous allons définir une application convenable de $I\left(J^{h} \mathscr{L}\right)$ sur $I(L)$ avec certaines propriétés.

LEMME 3.1. Soit $R^{m} \in I\left(J^{h} \mathscr{L}\right)$; alors, on a:

(a) $\left[\left(\underline{R^{m}}\right)^{+1}, \underline{J^{m+1} \mathscr{L}}\right]^{\prime} \subset \underline{R^{m}}$.

(b) $\left[\left(\underline{R^{m}}\right)^{+\ell+1}, \underline{J^{m+\ell+1} \mathscr{L}}\right]^{\prime} \subset\left(\underline{R^{m}}\right)^{+\ell}$, pour $\ell \geq 0$.

Preuve. La partie (a) découle de $\left[\underline{R^{m}}, \underline{J^{m} \mathscr{L}}\right] \subset \underline{R^{m}}$. Supposons:

$$
\left[\left(\underline{R^{m}}\right)^{+\ell+1}, \underline{J^{m+\ell+1} \mathscr{L}}\right]^{\prime} \subset\left(\underline{R^{m}}\right)^{+\ell},
$$

et soient $\xi^{m+\ell+2}, \eta^{m+\ell+2}$ et $Z$ des sections de $\left(R^{m}\right)^{+\ell+2}, J^{m+\ell+2} \mathscr{L}$ et de $\mathscr{L}$. Le Lemme 1.1 entraîne:

$$
D\left(\left[\xi^{m+\ell+2}, \eta^{m+\ell+2}\right]^{\prime}\right)(Z)=\left[D\left(\xi^{m+\ell+2}\right)(Z), \eta^{m+\ell+1}\right]^{\prime}+\left[\xi^{m+\ell+1}, D\left(\eta^{m+\ell+2}\right)(Z)\right]^{\prime},
$$

où $\eta^{m+\ell+1}=\Pi\left(\eta^{m+\ell+2}\right)$ et $\xi^{m+\ell+1}=\Pi\left(\xi^{m+\ell+2}\right) \in\left(R^{m}\right)^{+\ell+1}$. Ainsi, $D$ envoie $\left[\left(\underline{R^{m}}\right)^{+\ell+2}, \underline{J^{m+\ell+2} \mathscr{L}}\right]^{\prime}$ dans $T^{*} \otimes\left(\underline{R^{m}}\right)^{+\ell}$. D'autre part, ce crochet se projecte dans $\left(\underline{R^{m}}\right)^{+\ell}$; d'où, 


$$
\left[\left(\underline{R^{m}}\right)^{+\ell+2}, \underline{J^{m+\ell+2} \mathscr{L}}\right]^{\prime} \subset\left(\underline{R^{m}}\right)^{+\ell+1}
$$

c.q.f.d.

Soit $R^{m} \in I\left(J^{h} \mathscr{L}\right), a \in M$ et $I=\lim _{\ell}\left(R_{a}^{m}\right)^{+\ell}$. Le lemme 3.1 implique que $I$ est un idéal fermé de l'algèbre formelle $L$ en $a$. Ainsi, nous considérons l'application:

$$
\begin{aligned}
I\left(J^{h} \mathscr{L}\right) & \rightarrow I(L) \\
R^{m} & \rightarrow I
\end{aligned}
$$

qui est surjective d'après le Corollaire 2.1. Soient $R^{m}$ et $S^{p}$ deux équations de Lie dans $I\left(J^{h} \mathscr{L}\right)$ ayant la même image dans $I(L)$. Supposons $m \geq p \geq h, m=p+\ell ; \lim _{s}\left(R_{a}^{m}\right)^{+s}=\lim _{s}\left(S_{a}^{p}\right)^{+s}$ implique $R_{a}^{m}=R_{a}^{p+\ell}$ $=\left(S_{a}^{p}\right)^{+\ell} ; R^{p+\ell}$ et $\left(S^{p}\right)^{+\ell}$ étant deux équations de Lie invariantes par $\Gamma$ on obtient $R_{X}^{p+\ell}=\left(S_{X}^{p}\right)^{+\ell}$ pour $X \in M$; d'où, $R^{m}=R^{p+\ell}=\left(S^{p}\right)^{+\ell}$. Nous avons ainsi le résultat suivant:

THÉORÈME 3.1. Soit $(\mathscr{L}, M)$ un pseudogroupe transitif infinitésimal sur $M, L$ son algèbre formelle en a et h l'entier tel que $J^{m+1} \mathscr{L}=\left(J^{m} \mathscr{L}\right)^{+1}$ pour $m \geq h$; alors il existe une application surjective de $I\left(J^{h} \mathscr{L}\right)$ sur $I(L)$ et si deux équations différentielles de $I\left(J^{h} \mathscr{L}\right)$ ont la même image l'une de ces équations est le prolongement de l'autre.//

Soit $F(L)$ l'ensemble des idéaux fermés de $L$ définis par un feuilletage dans $\left(L, L_{0}\right)$ et $F\left(J^{h} \mathscr{L}\right)$ l'ensemble des équations de Lie $R^{m}$ de $I\left(J^{h} \mathscr{L}\right)$ pour lesquelles il existe un sous-fibré vectoriel $E$ de $T(M)$ tel que:

$$
\begin{aligned}
& {[\underline{E}, \mathscr{L}] \subset \underline{E} ; \text { Ceci implique }[\underline{E}, \underline{E}] \subset \underline{E} .} \\
& \Pi_{0}\left(R^{m}\right)=E \\
& R_{a}^{\infty}=\lim _{\ell}\left(R_{a}^{m}\right)^{+\ell}=L \cap J_{a}^{\infty} E .
\end{aligned}
$$

Le Théorème 2.1 (5) et le Corollaire 2.1 impliquent:

Proposition 3.1. Soit $(\mathscr{L}, M)$ un pseudogroupe de Lie infinitésimal transitif sur $M$ et $a \in M$. L étant l'algèbre formelle de $\mathscr{L}$ en a l'application $I\left(J^{\mathscr{L}} \mathscr{L}\right) \rightarrow I(L)$ induit une application suriective de $F\left(J^{h} \mathscr{L}\right)$ sur $F(L)$.

$\S 4$.

Soit $(\mathscr{L}, M)$ un pseudogroupe de Lie infinitésimal transitif sur $M$, 
$a \in M$ et $L=\lim _{h} J_{a}^{h} \mathscr{L}$ son algèbre formelle en $a$.

Considérons $I$ un idéal fermé de $L$ et posons $W^{h}=I / I^{h}$ pour $h \geq 0$. On a évidemment les propriétés:

$$
\begin{gathered}
W^{0} \subset V=T_{a}(M), \quad \Pi: W^{h} \rightarrow W^{h-1} \quad \text { est surjective }, \\
{\left[W^{h+1}, J_{a}^{h+1} \mathscr{L}\right]^{\prime} \subset W^{h} \quad \text { pour } h \geq 0 .}
\end{gathered}
$$

Dénotons par $p^{h}$ le noyau de $W^{h} \rightarrow W^{h-1}$. On a $p^{h+1} \subset\left(p^{h}\right)^{+1}$ pour $h \geq 1$.

Lemme 4.1. Soient I et $J$ deux idéaux fermés de $L$ et $h \geq 1$ tel que:

(1) $q^{m+1}=p^{m+1}=\left(p^{m}\right)^{+1} \quad$ pour $m \geq h$.

(2) $W^{m}=U^{m} \quad$ pour $0 \leq m \leq h$.

Alors il existe un isomorphisme de $L$ sur $L$ transportant $I$ sur $J$.

Preuve. Pour $m \geq 0, f \in \Gamma, f(a)=a$ induit un isomorphisme linéaire :

$$
\begin{aligned}
f^{m}: J_{a}^{m} \mathscr{L} & \rightarrow J_{a}^{m} \mathscr{L} \\
j_{a}^{m} X & \rightarrow j_{a}^{m} f^{*}(X)
\end{aligned}
$$

tel que, $\left[f^{m+1}(X), f^{m+1}(y)\right]^{\prime}=f^{m}[X, y]^{\prime}$; d'autre part, l'inclusion $\left[W^{m+1}\right.$, $\left.J_{a}^{m+1} \mathscr{L}\right]^{\prime} \subset W^{m}$ implique $f^{m}\left(W^{m}\right)=W^{m}$ pour $m \geq 0$.

Considérons le diagramme:

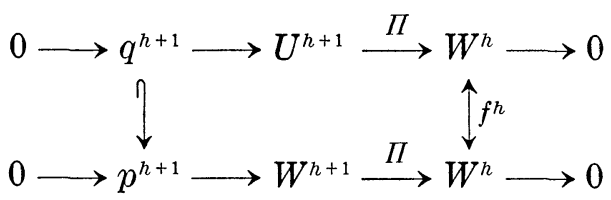

Montrons que $f^{h+1}\left(U^{h+1}\right)=W^{h+1}$. Soient $\alpha$ et $\beta$ des sections de $W^{h+1} \rightarrow W^{h}$ et $W^{h} \rightarrow U^{h+1}$ et définissons $\varphi: W^{h} \rightarrow J_{a}^{h+1} \mathscr{L} \operatorname{par} \varphi(X)=\left(f^{h+1} \circ \beta\right)$ $(X)-\left(\alpha \circ f^{h}\right)(X)$ alors,

$$
\Pi(\varphi(X))=f^{h}((\Pi \circ \beta)(X))-f^{h}(X)=f^{h}(X)-f^{h}(X)=0 ; \operatorname{donc} \varphi(X) \in g^{h+1}
$$

pour $X \in W^{h}$. En plus, $\delta(\varphi(X)) \in V^{*} \otimes g^{h}$ est tel que :

$$
\begin{aligned}
\delta(\varphi(X))(v) & =\left[\varphi(X), f^{h+1}(y)\right]^{\prime}=\left[f^{h+1}(\beta(X)), f^{h+1}(y)\right]^{\prime}-\left[\alpha\left(f^{h}(X)\right), f^{h+1}(y)\right]^{\prime} \\
& =f^{h}[\beta(X), y]^{\prime}-\left[\alpha\left(f^{h}(X)\right), f^{h+1}(y)\right]^{\prime}
\end{aligned}
$$

appartient à $W^{h}$, où $v \in V$ est la projection de $f^{h+1}(y)$ pour y dans $J_{a}^{h+1} \mathscr{L}$. Ainsi, $\delta(\varphi(X)) \in V^{*} \otimes p^{h}$. Par hypothèse, $\varphi(X)$ est dans $p^{h+1}=\left(p^{h}\right)^{+1}$. On a donc une application $\varphi: W^{h} \rightarrow p^{h+1}$ telle que $f^{h+1}(\beta(X))=\varphi(X)$ 
$+\alpha\left(f^{h}(X)\right)$ pour $X \in W^{h}$; alors $f^{h+1}\left(U^{h+1}\right) \subset W^{h+1}$, car $q^{h+1}=p^{h+1}$. De

$$
\begin{aligned}
\operatorname{dim} W^{h+1} & =\operatorname{dim} W^{h}+\operatorname{dim} p^{h+1}=\operatorname{dim} W^{h}+\operatorname{dim} q^{h+1} \\
& =\operatorname{dim} U^{h+1}=\operatorname{dim} f^{h+1}\left(W^{h+1}\right),
\end{aligned}
$$

on obtient,

$$
f^{h+1}\left(U^{h+1}\right)=W^{h+1} \text {. De } f^{h+1}\left(U^{h+1}\right)=U^{h+1} \text { on obtient, } W^{h+1}=U^{h+1} \text {. }
$$

Un raisonnement analogue prouve que $f^{m}\left(W^{m}\right)=U^{m}$ pour $m \geq h+1$; ainsi $\varphi=\lim _{m} f^{m}$ est un isomorphisme de $L$ sur $L$ qui envoie $I$ sur $J$.

c.q.f.d.

Définition 4.1. Un ensemble $W^{h}=\left\{W^{0}, W^{1}, W^{2}, \cdots, W^{h}\right\}$ d'espaces vectoriels tel que:

(1) $W^{0} \subset V=T_{a}(M)$.

(2) $W^{m} \subset J_{a}^{m} \mathscr{L}$ pour $m \geq 1$ et $m \leq h$.

(3) $\left[W^{m+1}, J_{a}^{m+1} \mathscr{L}\right]^{\prime} \subset W^{m}$, pour $0 \leq m<h-1$.

(4) La projection $\Pi: W^{m+1} \rightarrow W^{m}$ est surjective pour $0 \leq m<h-1$. sera dite une algèbre de Lie normale tronquèe d'ordre $h \geq 1$.

Pour $m \leq h$ dénotons par $p^{m}$ le noyau de la projection $\Pi: W^{m}$ $\rightarrow W^{m-1}$; pour $m \geq h+1$ posons $p^{m+1}=\left(p^{m}\right)^{+1}$. Dénotons par $H^{j, m}\left(W^{h}\right)$ la cohomologie de Spencer du complexe:

$$
\bigwedge^{j-1} V^{*} \otimes p^{m+1} \stackrel{\delta}{\longrightarrow} \bigwedge^{j} V^{*} \otimes p^{m} \stackrel{\delta}{\longrightarrow} \bigwedge^{j+1} V^{*} \otimes p^{m-1}
$$

associé à $\left\{p^{m}\right\}_{m \geq 1}$.

Lemme 4.2. Pour $h \geq 1$ soit $W^{h}=\left\{W^{0}, W^{1}, \cdots, W^{h}\right\}$ une algèbre de Lie normale tronquée d'ordre $h$ telle que, $H^{1, h-1}\left(W^{h}\right)=H^{2, h-1}\left(W^{h}\right)=0$. Supposons $g^{h+1}=\left(g^{h}\right)^{h+1}$; alors il existe un sous-espace vectoriel $W^{h+1}$ $\subset J_{a}^{h+1} \mathscr{L}$ tel que $W^{h+1}=\left\{W^{0}, W^{1}, \cdots, W^{h+1}\right\}$ est une algèbre de Lie normale tronquée d'ordre $h+1$ et $p^{h+1}$ est $\operatorname{Ker}\left\{W^{h+1} \rightarrow W^{h}\right\}$.

Preuve. Considérons les complexes:

$$
\begin{aligned}
& 0 \longrightarrow p^{h+1} \stackrel{\delta}{\longrightarrow}\left(J_{a}^{h} \mathscr{L}\right)^{*} \otimes p^{h} \stackrel{\delta}{\longrightarrow} \bigwedge^{2}\left(J_{a}^{h} \mathscr{L}\right)^{*} \otimes p^{h-1} \\
& \stackrel{\delta}{\longrightarrow} \Lambda^{3}\left(J_{a}^{h} \mathscr{L}\right)^{*} \otimes p^{h-2}
\end{aligned}
$$

et 


$$
0 \longrightarrow g^{h+1} \stackrel{\delta}{\longrightarrow}\left(J_{a}^{h} \mathscr{L}\right)^{*} \otimes g^{h} \stackrel{\delta}{\longrightarrow} \wedge^{2}\left(J_{a}^{h} \mathscr{L}\right)^{*} \otimes g^{h-1}
$$

Les hypothèses impliquent (1) et (2) exactes. Pour définir $W^{h+1}$ nous allons construire une section $\gamma: W^{h} \rightarrow J_{a}^{h+1} \mathscr{L}$ telle que $\left[\gamma\left(W^{h}\right), J_{a}^{h+1} \mathscr{L}\right]^{\prime} \subset W^{h}$. Ainsi $W^{h+1}=\operatorname{im} \gamma+p^{h+1}$ vérifie le lemme.

Pour $X \in W^{h}$ posons $\varphi_{X}(y)=\mathscr{L}[X, y]^{\prime}-[\beta(X), \beta(y)]^{\prime}$ pour $y \in J_{a}^{h} \mathscr{L}$ où $\alpha$ et $\beta$ sont des sections de $W^{h} \rightarrow W^{h-1}$ et $J_{a}^{h+1} \mathscr{L} \rightarrow J_{a}^{h} \mathscr{L}$; alors, $\Pi\left(\varphi_{X}(y)\right)$ $=[X, y]^{\prime}-[\Pi \beta(X), \Pi \beta(y)]^{\prime}=0$, d'où, $\varphi_{X}$ est à valeurs dans $g^{h}$. En plus, pour $y, z$ dans $J_{a}^{h} \mathscr{L}$ on a:

$$
\left(\delta \varphi_{X}\right)(y, z)=\left[\varphi_{X}(z), y\right]^{\prime}-\left[\varphi_{X}(y), z\right]^{\prime}=\left[\alpha[y, z]^{\prime}, X\right]^{\prime}-\left[[\beta(y), \beta(z)]^{\prime}, X\right]^{\prime} .
$$

Ainsi, $\delta \varphi_{X}: \bigwedge^{2}\left(J^{h} \mathscr{L}\right) \rightarrow p^{h-1}$; de $\delta \delta \varphi_{X}=0$ on a l'existence d'une application $\psi_{X}: J_{a}^{h} \mathscr{L} \rightarrow p^{h}$, telle que $\delta \psi_{X}=\delta \varphi_{X}$. Ceci implique que $\varphi_{X}-\psi_{X}$ de $J_{a}^{h} \mathscr{L}$ dans $g^{h}$ est un cocycle; de $g^{h+1}=\left(g^{h}\right)^{+1}$ on obtient un élément $\eta_{X} \in g^{h+1}$ tel que $\delta \eta_{X}=\varphi_{X}-\psi_{X} ;$ d'où, l'existence d'une application linéaire $\eta: W^{h} \rightarrow g^{h+1}$ telle que $\eta(X)=\eta_{X}=\varphi_{X}-\psi_{X}$. Posons $\gamma=\beta+\eta$; alors, pour $X \in W^{h}$ et $y \in J_{a}^{h+1} \mathscr{L}$ on obtient:

$$
\begin{aligned}
{[\gamma(X), y]^{\prime} } & =[\eta(X)+\beta(X), y]^{\prime}=\left(\varphi_{X}-\psi_{X}\right)(\Pi(y))^{\prime}+[\beta(X), y]^{\prime} \\
& =\alpha\left([X, \Pi(y)]^{\prime}\right)-[\beta(X), \beta(\Pi(y))]^{\prime}-\left[\psi_{X}, \Pi(y)\right]^{\prime}+[\beta(X), y]^{\prime} \\
& =\mathscr{L}\left([X, \Pi(y)]^{\prime}\right)-\left[\psi_{X}, \Pi(y)\right]^{\prime}+[\beta(X), y-\beta \Pi(y)]^{\prime}
\end{aligned}
$$

qui est dans $W^{h}$; d'où, $\left[\operatorname{im} \gamma, J_{a}^{h+1} \mathscr{L}\right]^{\prime} \subset W^{h}$; ceci entraîne notre résultat.

c.q.f.d.

THÉORÈME 4.1. Soit $(\mathscr{L}, M)$ un pseudogroupe de Lie infinitésimal transitif sur $M, L$ son algèbre formelle en $a \in M$ et $W^{h}=\left\{W^{0}, W^{1}, \ldots\right.$, $\left.W^{h}\right\}$ une algèbre de Lie normale tronquée d'ordre $h \geq 1$ telle que $H^{1, m}$ $=H^{2, m}\left(W_{h}\right)=0$, pour $m \geq h-1$. Supposons $g^{m+1}=\left(g^{m}\right)^{+1}$ pour $m \geq h$; alors il existe un idéal fermé $I$ de $L$ ayant $W^{h}$ comme algèbre tronquée d'ordre $h$. En plus, si $J$ est un autre idéal de $L$ d'algèbre tronquée $W^{h}$, alors $i l$ existe un isomorphisme de $L$ transportant $I$ sur $J$ si $q^{m+1}$ $=p^{m+1}$ pour $m \geqslant h$.

Preuve. Ces résultats découlent du Lemme 4.2 et du Lemme 4.1.

c.q.f.d.

\section{REFERENCES}

[1] Isao Hayashi, Embedding and Existence theorems of infinite Lie algebra, J. Math. 
Soc. Japan, Vol. 22, 1970.

[2] H. Goldschmidt, Sur la structure des équations de Lie III, La cohomologie de Spencer, J. of Diff. Geometry, Vol. 11, No. 2, 1976.

[ 3 ] Pierre Molino, Théorie des G-Structure: Le problème d'équivalence, Lectures Notes in Mathematics, Springer Verlag, No. 588, 1977.

[4] Ngo Van Quê et A. A. M. Rodriguês, Troisième théorème fondamental de réalisation de Cartan, Annales de l'Institut Fourier (Grenoble), 1975.

Universite des Sciences and Techniques

du Languedoc

Mathématiques-Montpellier 